\title{
SURGICAL ASPECTS OF DISSECTING AORTIC ANEURYSMS*
}

FRANCIS S. GERBASI, M.D.

Long Beach, California

AND

ALEXANDER BLAIN III, M.D.

Detroit, Michigan

Great progress has been made in recent years in the surgical treatment of syphilitic and arteriosclerotic aneurysms as well as other lesions of the aorta $(1,2)$, but very little advance has been made in the treatment of dissecting aneurysms. We have been led to hope that the time has arrived to suggest that surgical techniques be developed to treat this disease. Such techniques are probably doomed to failure, however, unless based on a knowledge of the pathogenesis of dissecting aneurysms.

A dissecting aneurysm is a lesion characterized by a hematoma which dissects the medial coats of the aorta. Frequently there is a rupture of the intima. Previously it was believed that the intimal rupture was the primary event in the pathogenesis of this disease. It is now known that intimal rupture is unnecessary in the production of dissecting aneurysms but that it may be a complicating factor (3).

Further evidence of this fact was substantiated by our attempts to produce dissecting aneurysms in rabbits and in ten dogs by incising the intima and media and separating these layers from the adventitia for a short distance. These attempts were unsuccessful and we were unable to produce a dissecting aneurysm. Both the incised intima and the space created between the media and adventitia filled with blood which clotted and organized.

These aneurysms result from medial hemorrhage due to ruptured vasa vasora. The cause of rupture of the vasa vasorum is not clearly understood but cystic medial degeneration and other processes producing ischemia have been implicated $(4,5,6)$. The hemorrhage which occurs causes pressure on the overlying intimal layer, weakening it to such an extent that it finally tears. This tear is usually transverse, clear cut, in the ascending aorta and not associated with a sclerotic plaque (7) (Fig. 1). Aortic blood now enters this rent in the intima causing further extension of the dissection. The dissecting hematoma may extend toward the heart or away from it or in both directions (Fig. 2). With extension distally the extravasating blood often involves many of the aortic branches, producing ischemia or infarction of the area supplied.

Clinical manifestations of this disease are protean and depend upon the extent of the dissection and the aortic branches involved. They have been discussed previously $(8,9,10)$ emphasizing the fact that this entity has been incorrectly

From the Departments of Surgery, The University of Michigan Hospital, Ann Arbor, Michigan and the Alexander Blain Hospital, Detroit, Michigan. 


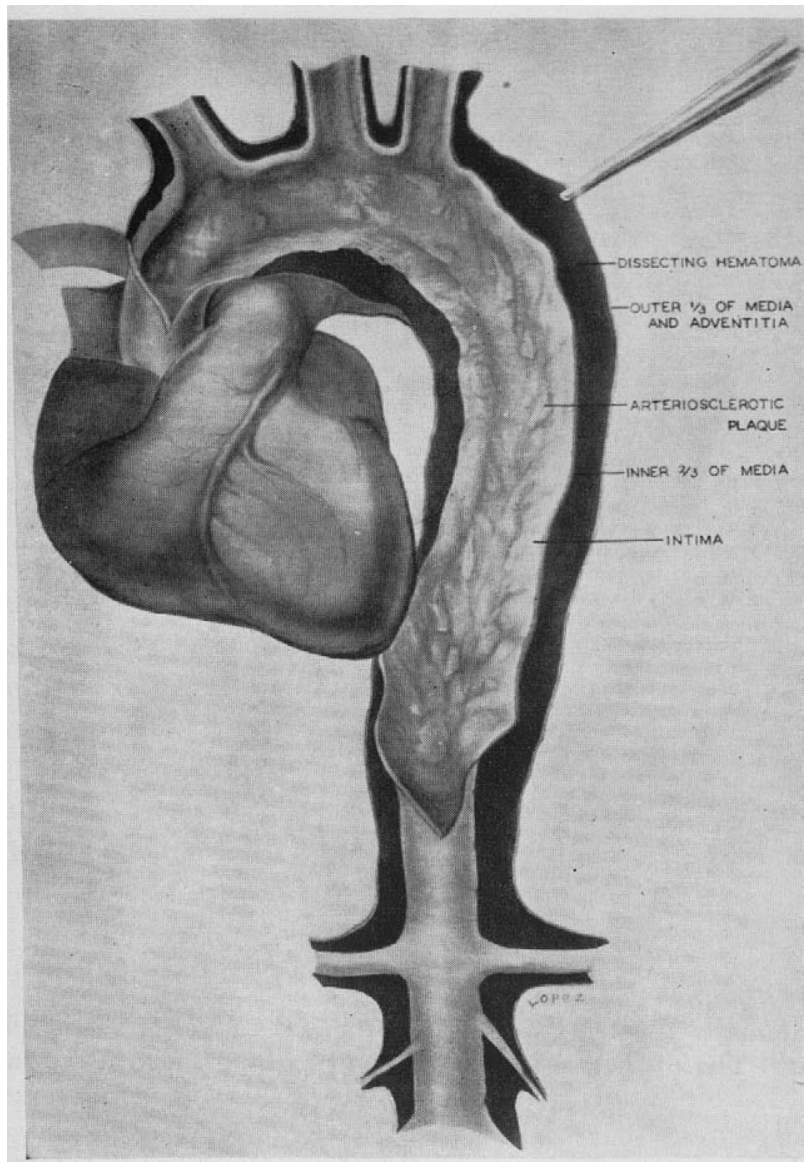

Fia. 1. Drawing illustrates a dissecting aneurysm without an intimal rupture. The hematoma is between the inner two-thirds and outer one-third of the media.

diagnosed as a surgical condition 12.9 per cent of the time and that one-half of the cases diagnosed as a surgical condition have had futile operative intervention. This is sufficient evidence to warrant surgeons' familiarity with the signs and symptoms of this disease. Also it should be re-emphasized, in order to justify the surgical attempts made to benefit these patients, that the result of such an aneurysm is almost uniformly rapidly fatal. The eventual outcome in 95 per cent of the cases is external rupture into the pericardium with resultant tamponade (70 per cent), or into the retropleural and retroperitoneal tissues with exsanguination. This usually occurs within two or three days (11). Rarely such an aneurysm will heal itself by rupturing back into the aortic lumen with the formation of a "double barreled" aorta as described by MacCallum.

One of the most interesting cases of dissecting aortic aneurysms ever reported was published by Golden and Weens of Emory University (12). In this case a definite diagnosis was made by angiocardiography which permitted accurate 


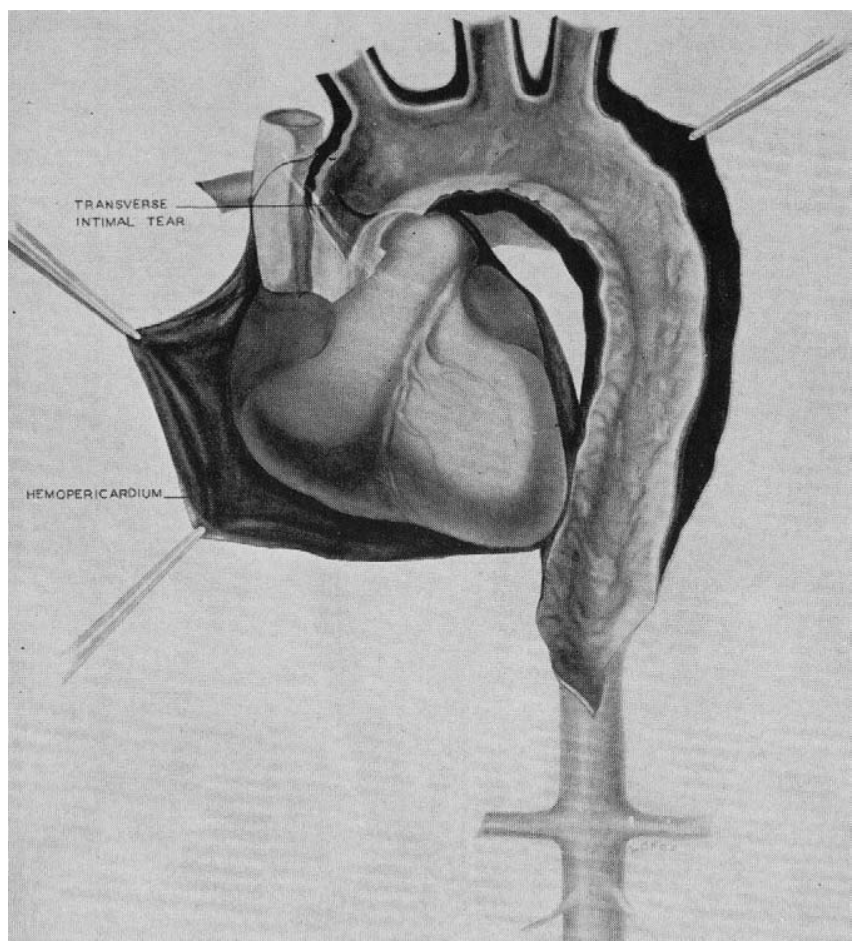

FIG. 2. A diagrammatic illustration of a hypothetical case of a dissecting aneurysm with an intimal tear. The dissection has progressed proximally rupturing into the pericardium.

demonstration of the size and extent of the dissection. Furthermore, this dissecting aneurysm was wrapped with cellophane by Dr. Osler Abbott and the patient was reported to be in good health five months later. This was, as far as we know, the first patient treated surgically who survived even the period of hospitalization.

\section{SURGICAL TECHNIQUES}

We wish to make clear that the following are suggestions and opinions and are not based upon an experience with the definitive surgical therapy of dissecting aneurysms. Rather, the following statements are based on an opportunity to study during life and at the autopsy table, several cases of this disease. This experience was afforded at the Johns Hopkins, University of Michigan and Alexander Blain Hospitals as well as by material from other institutions. We have interested ourselves in this subject, particularly its surgical aspects, for the past ten years.

Cases of dissecting aneurysms with rupture into the pericardium, producing the signs and symptoms of acute cardiac tamponade, should have immediate aspiration of the pericardial sac while the operating room is being prepared 
for an emergency thoracotomy. The left chest should be entered through the fourth left anterior intercostal space, dividing the sternum transversely. The pericardium and adventitia of the ascending aorta are incised longitudinally releasing the tamponade. The ascending aorta is immediately inspected as this is the most common site for an intimal tear, if present. The bleeding may be reduced by placing a finger over the rent, or if this is not possible, the area may be packed with Gel Foam or an excised piece of muscle. $A$ few sutures are placed about the tear and tied, thus approximating the torn edges of the intima and media and also holding the Gel Foam or muscle in place.

Case report. Mr. I. N. (No. 735550 Univ. of Mich.), this sixty-four year old white man was operated upon on September 24,1952 by one of us (F. S. G.) with a preoperative diagnosis of a dissecting aneurysm with progression down the aorta. A left thoracotomy was done in the hope that a definitive surgical procedure could be accomplished. A greatly dilated, arteriosclerotic aortic arch was found. There was no evidence of aneurysmal dissection; however, the origin of the aorta from the heart was not closely inspected. It was felt that the diagnosis was incorrect and therefore the chest was closed after injecting the adventitial wall of the arch of the aorta with 0.9 per cent dicetyl phosphate in olive oil. The patient died 12 hours after operation. At autopsy a dissecting aneurysm beginning in the proximal half of the ascending aorta, progressing toward the heart, and rupturing into the pericardium was found. Undoubtedly this dissecting hematoma was present at the time of exploration but had not as yet ruptured into the pericardium. If this had been recognized perhaps death due to acute cardiac tamponade could have been averted. In another case one of us (A. B. III) operated upon a patient with a dissecting aortic aneurysm which had ruptured into the retroperitoneal tissue (Case No. A. B. H. 51-1375). During the operation the patient went into shock and died on the operating table before any definitive procedure could be accomplished.

Cases in which the aneurysm has not ruptured intrapericardially but has progressed down the descending aorta afford a slightly different problem. A left thoracotomy incision should be made through the fourth anterior interspace and the aorta inspected. The extent of the dissection should be determined. In the majority of cases an intimal rupture will be present. If the patient's condition is satisfactory, the adventitia and outer one-third of the media are incised over the area of the suspected intimal rupture (usually over the ascending aorta). The extravasated blood in the area is quickly removed and the bleeding controlled, as already mentioned, by placing a finger, Gel Foam or a free muscle graft over the rent. Interrupted sutures should be placed on both sides of the tear and tied as the finger is withdrawn from the area. Following this maneuver a few small incisions are made in the outer one-third of the media and adventitia of the remaining portion of the aorta where the dissection has extended. The extravasated blood is aspirated and Gel Foam packed about the aorta in the plane where the dissection had occurred (Fig. 3, $A$ ). This should stop further bleeding from the vasa vasorum. It would then seem logical to attempt to wrap the aorta with cellophane, fascia, wire mesh or other substance. 


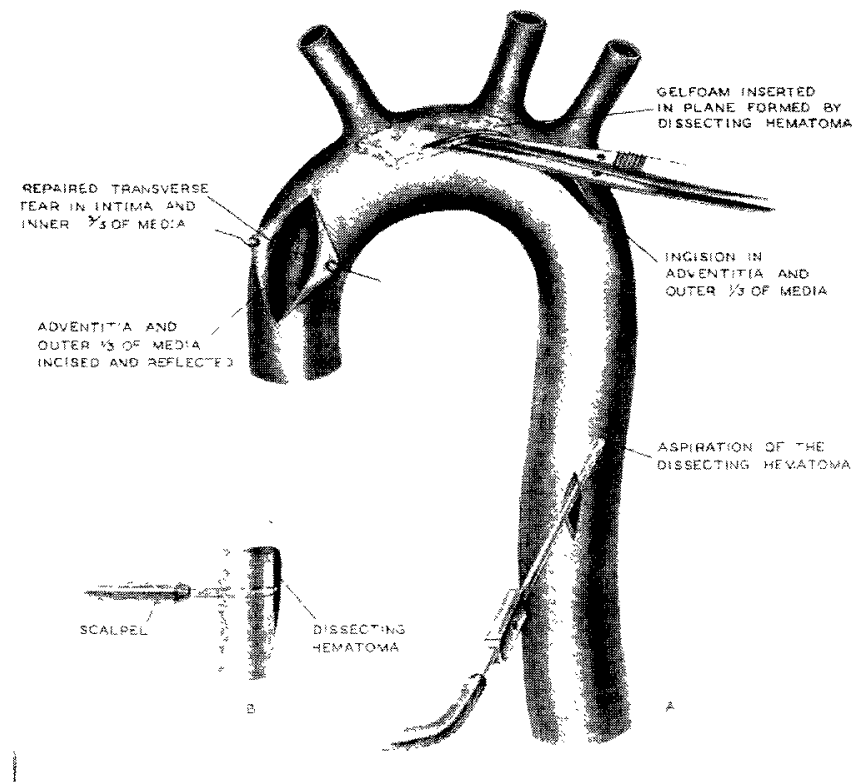

Fra. 3. Proposed methods of repair. A. Showing incised and reflected adventitia and outer one-third of media over the site of a transverse tear which has been repaired with interrupted silk sutures. Gel Foam is shown being inserted in the cleavage plane formed by the dissecting hematoma. $B$. Demonstration of the method to produce a "double-barreled" aorta by inserting a long, thin blade knife through the aortic lumen and opposite intima, thus allowing the hematoma to rerupture back into the lumen.

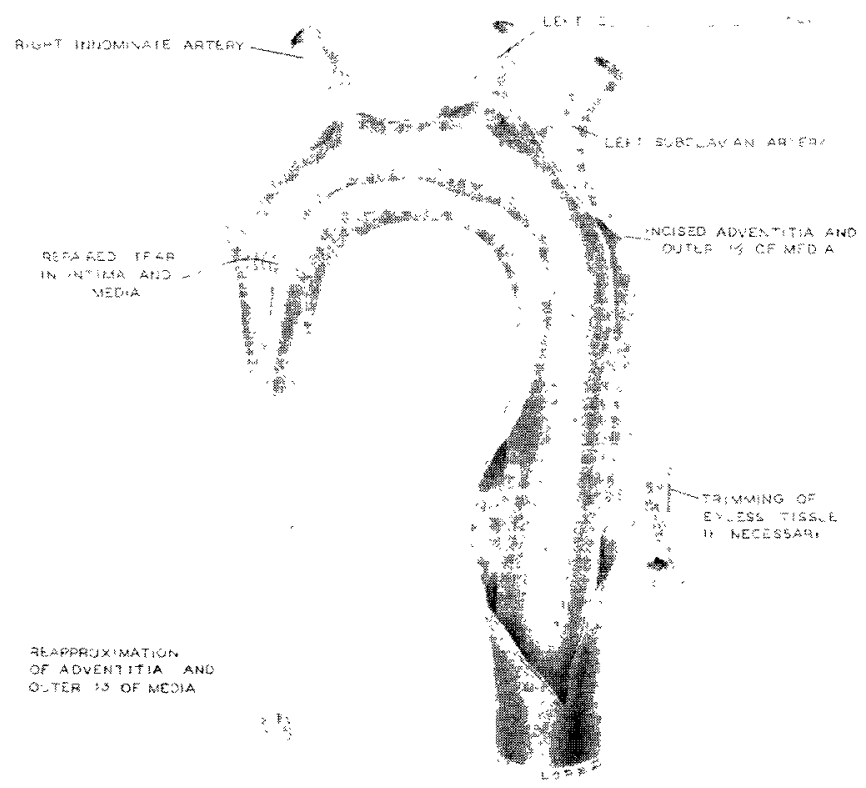

Fig. 4. A method of repair which can be used with or without an intimal tear, preferably in a patient having little or no evidence of intimal sclerosis. 
In cases in which no tear in the aortic intima can be found, or very little atherosclerosis seen, one could completely incise the outer onc-third of the media and adventitia, aspirate the blood, place a layer of Gel Foam in the cleavage plane and then approximate the incised edges of the media and adventitia (Fig. 4). This method could also be used in cases with intimal tears, especially in persons less than forty years of age, who usually have very little evidence of mitimal sclerosis. Of course, the patient's condition must be such as to tolerate this longer procedure.

\section{DISCUSSION}

Many cases of dissecting aneurysms have been diagnosed as "acute surgical abdomen" and have had abdominal exploration. If a surgeon finds that he is dealing with a dissecting aneurysm, the adventitia and media should not be incised at this time, for the great majority of the dissections begin in the upper thoracic aorta and are associated with a rupture of the intima in the ascending aorta. If the hematoma were released, the source of the bleeding could not be reached and the patient might become exsanguinated. Therefore, if the patient's condition is good, the abdomen should be closed, the left chest opened and one of the procedures suggested in this article carried out. If the patient's condition is too precarious for the addition of a thoracotomy, an attempt can be made to establish a double-barreled aorta by making a stab wound through the portion of the abdominal aorta where thes dissection has not occurred, and extend the knife through the aortic lumen and opposite intima and inner two-thirds of the media so that the end of the blade is in the lower edge of the dissecting hematoma (Fig. 3, B). We are not sure this will succeed but we do know that patients who survive an acute dissecting aneurysm often do so because the blood has re-entered the aortic lumen by intimal rupture. Such an incision is a simple procedure and should not take more than a few minutes. If such a procedure were carried out and were successful the prognosis would not be good, since it is known that patients whose dissecting hematoma has ruptured into the aorta, have had a subsequent external rupture from two weeks to two years after the first onset of symptoms (6). Patients in whom a double-barreled aorta has been surgically created should have a wrapping of the aorta or injection of dicetyl phosphate in the adventitial wall.

If a dissecting aneurysm is limited to the abdominal aorta, which is relatively uncommon, one should incise the outer one-third of the media and adventitia, release the hematoma and close the rent in the intima and inner two-thirds of the media by suturing it together, if possible, or pack it with some hemostatic material. Johns recently carried out this procedure and, although the patient died seven days postoperatively of renal insufficiency, he was able to repair the rent in the aorta and prevent further dissection and external rupture (13).

If severe hypertension exists, a left dorsal sympathectomy should be performed before closing the chest. Also, one should wrap the aorta with polythene or inject dicetyl phosphate into the adventitia of the vessel to prevent the likelihood of external rupture. 
Considering the severity of this disease, the precarious condition of so many patients who are afflicted with it, and the fact that the ascending and transverse portions of the aorta are usually involved, we feel that resection of the involved aorta and restoration of aortic continuity with a graft is not feasible.

\section{SUMMARY}

The treatment of dissecting aneurysms by the conventional conservative methods has only infrequently prolonged life. We believe that surgical intervention as outlined may prolong the lives of some of these patients. We realize that the suggested procedures are not supported by experimental studies, since the difficulty in producing the lesions experimentally has not yet been overcome. Based on present knowledge of the pathogenesis of dissecting aneurysms, the techniques described seem reasonable, logical and worthy of trial.

2201 Jefferson Ave. East. Detroit 7 , Mich.

\section{BIBLIOGRAPITY}

1. Cowley, R. A., Sloan, H. E. And Sullenberger, N. H.: Successful Repair of an Aortic Ancurysm with Sternal Perforation. J. Thoracic Surg. 21: 159, 1951.

2. Cooley, D. A. AND DeBaKey, M. E.: Surgical Considerations of Intrathoracic Aneurysms of the Aorta and Great Vessels. Ann. Surg. 135: 660, 1952.

3. Blain, A. III: Dissecting Aortic Aneurysms without Intimal Rupture: A Note on Pathogenesis of Dissecting Aneurysms, Univ. Hosp. Bull., Ann Arbor, 13: 21, 1947.

4. Robmetson, J. S. ANo Smrth, K. V.: An Analysis of Certain Factors Associated with the Production of Fxperimental Dissection of the Aorta Media, in Relation to the Pathogenesis of Dissecting Aneurysms, Jour. Path. and Bact., 60: 43, 1948.

5. Schlicter, J. G., Amdromin, G. D. and Solway, A. J. L.: Dissecting Aneurysm of the Aorta, Arch. Int. Med., 84: 558, 1949.

6. Gone, I.: Pathogenesis of Dissecting Aneurysm of the Aorta, A.M.A. Arch. Path., 53: $142,1952$.

7. Whiss, S.: Spontaneous Dissecting Aneurysms of the Aorta, M. Clin. North America, 18: $1117,1935$.

8. Blain, A. III and Gerbasi, F. S.: The Surgical Significance of Dissecting Aortic Aneurysms, Surgery, 25: 628, 1949.

9. Gerbasi, F. S., Woodburne, H. L. and Hea vener, L. E. : Dissecting Aortic Aneurysm. A Consideration of the Clinieal Manifestations and Report of a Case Diagnosed Ante Mortem, Alexander Blain Hosp. Bull., 6: 57, 1947.

10. Blain, A. III, Glynn, T. P. ANı Hinatzka, T.: Dissecting Aortic Aneurysms Involving a Renal Artery and Simulating $\Lambda$ cute Nephrolithiasis, J. Urol., 63: 753, 1945.

11. Leitch, W. H.: Dissecting Ancurysm With Case Reports, Bull. School Med. Univ., Maryland, 29: 7, 1944.

12. Golden, A. And Werns, H. S.: The Diagnosis of Dissecting Aneurysms of the Aorta by Angiocardiography, Report of a Case, Am. Heart Jour., 37: 114, 1949.

13. Johns, T. N. P.: Dissecting Aneurysm of the Abdominal Aorta, Report of a Case with Repair of Perforation, Ann. Surg., 137: 232, 1953. 